\title{
Nano-based chemical sensor array systems for uninhabited ground and airborne vehicles
}

Christina Brantley, Paul Ruffin, Eugene Edwards

Christina Brantley, Paul B. Ruffin, Eugene Edwards, "Nano-based chemical sensor array systems for uninhabited ground and airborne vehicles," Proc. SPIE 7291, Nanosensors, Biosensors, and Info-Tech Sensors and Systems 2009, 729102 (31 March 2009); doi: 10.1117/12.820760 Health Monitoring, 2009, San Diego, California, United States 


\title{
Nano-based Chemical Sensor Array Systems for Uninhabited Ground and Airborne Vehicles
}

\author{
Christina Brantley, Paul B. Ruffin, Eugene Edwards \\ U. S. Army Research, Development, and Engineering Command \\ ATTN: AMSRD-AMR-WD \\ Redstone Arsenal, Alabama 35898 \\ E-mail: christina.brantley@us.army.mil
}

\begin{abstract}
In a time when homemade explosive devices are being used against soldiers and in the homeland security environment, it is becoming increasingly evident that there is an urgent need for high-tech chemical sensor packages to be mounted aboard ground and air vehicles to aid soldiers in determining the location of explosive devices and the origin of biochemical warfare agents associated with terrorist activities from a safe distance. Current technologies utilize relatively large handheld detection systems that are housed on sizeable robotic vehicles. Research and development efforts are underway at the Army Aviation \& Missile Research, Development, and Engineering Center (AMRDEC) to develop novel and less expensive nano-based chemical sensors for detecting explosives and chemical agents used against the soldier. More specifically, an array of chemical sensors integrated with an electronics control module on a flexible substrate that can conform to and be surface-mounted to manned or unmanned vehicles to detect harmful species from bio-chemical warfare and other explosive devices is being developed. The sensor system under development is a voltammetry-based sensor system capable of aiding in the detection of any chemical agent and in the optimization of sensor microarray geometry to provide nonlinear Fourier algorithms to characterize target area background (e.g., footprint areas). The status of the research project is reviewed in this paper. Critical technical challenges associated with achieving system cost, size, and performance requirements are discussed. The results obtained from field tests using an unmanned remote controlled vehicle that houses a $\mathrm{CO}_{2} /$ chemical sensor, which detects harmful chemical agents and wirelessly transmits warning signals back to the warfighter, are presented. Finally, the technical barriers associated with employing the sensor array system aboard small air vehicles will be discussed.
\end{abstract}

Keywords: Micro-sensor arrays, voltammetry-based sensor system, bio-chemical warfare agents

\section{INTRODUCTION}

In the Global War on Terrorism climate, military personnel and civilians are being faced with the problem of uncertain attacks due to hidden explosive devices and chemical warfare agents on public terrain, as well as on the battlefield. High-tech chemical sensor packages are being sought to be mounted aboard small autonomous uninhabited vehicles (AUV) to detect and track chemical plumes and keep the soldiers out of harm's way.

Commercially available chemical sensors, which can be mounted onto airborne and ground vehicles, are typically large, heavy, expensive, and sensitive to a single or a limited number of chemical species. ${ }^{1,2,3}$ General Atomics has developed smart micro-sensors for the Naval Research Laboratory (funded by the Office of Naval Research) to detect shipboard fires. ${ }^{4}$ The Joint Chemical Agent Detector (JCAD) ${ }^{5}$ is a handheld chemical agent point detector for monitoring chemical warfare agents to facilitate situational awareness in a hostile chemical environment. The aforementioned sensor types do not meet the size and power requirements for use on miniature uninhabited vehicles.

Rapid advances in micro- and nanotechnology are fueling the development of a new generation of miniature chemical sensors. Sandia National Laboratory has developed a nano-enabled chemical sensor that is integrated into a micro uninhabited aerial vehicle (UAV). ${ }^{6}$ Georgia Tech has developed an half-pound chemical sensor that is mounted in the nose cone of a mini-UAV. ${ }^{7}$ Efforts are underway to reduce the weight to one ounce for wing mounting. These

Nanosensors, Biosensors, and Info-Tech Sensors and Systems 2009, edited by Vijay K. Varadan, Proc. of SPIE Vol. 7291, 729102 - (c) 2009 SPIE · CCC code: 0277-786X/09/\$18 · doi: 10.1117/12.820760 
miniature chemical sensors are sensitive to a limited number of chemical species. To date, there has not been a report of ultra-small, low power chemical sensors that can sense a wide variety of chemical species.

The Army Aviation and Missile Research, Development, and Engineering Center (AMRDEC), in collaboration with Alabama A\&M University and Streamline Automation LLC, is conducting a research and development program to demonstrate low power, ultra-miniature sensor systems that can detect a wide variety of electro-active chemical species in the parts-per-billion range. The primary objective of the project is to advance the proven voltammetry-based sensing technique via adopting microlithography for the fabrication of miniaturized sensor array packages, which can be mounted aboard micro-unmanned autonomous vehicles for detecting and identifying a wide variety of unidentified analytes. The ultra-miniature sensor system can be coupled with a GPS Receiver to predict the location of explosive devices and/or chemical plumes.

This paper is intended to present the status of the AMRDEC research and development project. The paper is arranged as follows. A brief discussion of the advanced voltammetry-based sensor system is provided in Section 2. The integration of votammetry-based sensor systems onto unmanned vehicles is presented in Section 3. Preliminary test results and data acquisition/analysis are discussed in Section 4. A summary is provided in Section 5.

\section{VOLTAMMETRY-BASED SENSOR SYSTEM}

The thick-film voltammetric sensor technology, originally developed by Argonne National Laboratory and licensed by Streamline Automation for use in the detection of gases, is adopted as the baseline for the AMRDEC research and development project. ${ }^{8,9}$ The thick-film sensor, as shown in Fig. 2.1-1, has a solid electrolyte with multiple electrodes for simultaneously sensing three different analytes or targeted chemical agents. Test results for the subsequent thick-film sensor design indicate that the sensor can successfully detect hypergolic propellants off-gassing.

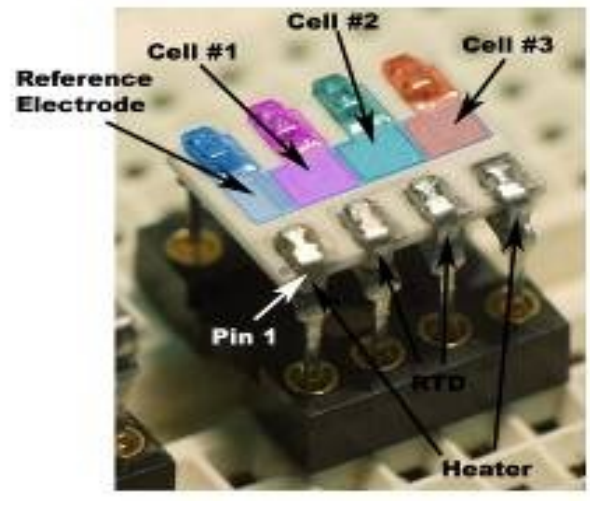

Fig. 2.1-1 Prototype of Triple Element Thick Film Sensor

The primary objective of the AMRDEC research and development project is to develop a miniaturized chemical sensor array package that can be mounted onto an uninhabited vehicle to detect and identify multiple analytes from explosive devices, as well as from chemical warfare agents. The sensor has to be ruggedized for military environments and miniaturized for minimal payload additions on uninhabited vehicles. The size, power consumption, and surface temperature dependence of the sensor are minimized by redesigning the sensor substrate of the thick-film device to a thin film configuration. Useful lessons learned from the thick-film design are utilized for the thin-film devices, which display the same electrochemical behavior as the thick film, especially when adequate attention is given to fabrication steps (such as cleaning, surface preparation, material purity, sputtering, target preparation, and control of process parameters during reactive magnetron sputtering). ${ }^{10}$ It should likewise be noted that the response and recovery speeds of thin-film devices are faster than similarly configured thick-film devices. 
Research on microelectrodes for gas sensors on bulk or thin-film substrates is extremely rare for voltammetric methods; however, all electrochemical methods share similar physics, differing mostly by the method of interrogation (passive or active) and variable measured (current or potential). ${ }^{11}$

\subsection{Design Approach}

A study of various geometrical configurations for the electrode deposition is conducted in order to generate a design layout for a thin-film sensor. Three configurations are investigated in order to determine the relative sensitivity of different sensing electrode designs and the response to the atomic structures of various chemical species. The resulting designs were the combed (A), solid (B), and grid (C) patterns, as shown in Fig. 2.1-2.

The combed design is selected for the thin-film sensor electrode deposition. The preliminary sensor design, as shown in Fig. 2.1-3, demonstrates the combed configuration. Each sensor has a reference electrode intertwined in a combed design with three different sensing electrodes, allowing for the detection of at least three different gas contaminants.
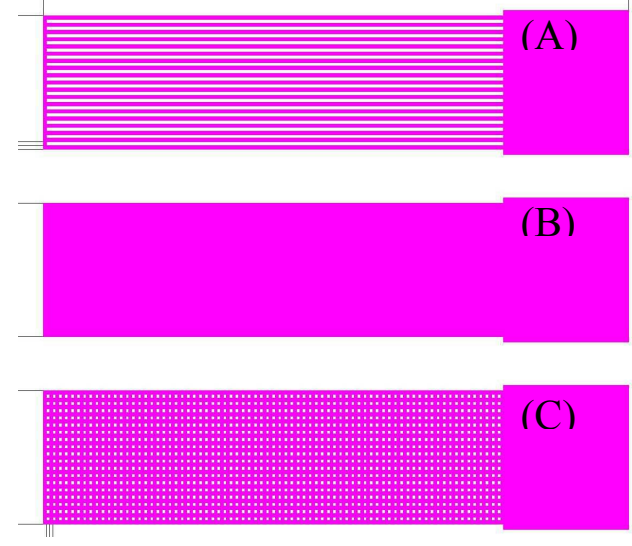

Fig. 2.1-2 Schematic of Sensor Describing the Test Electrolyte Deposition

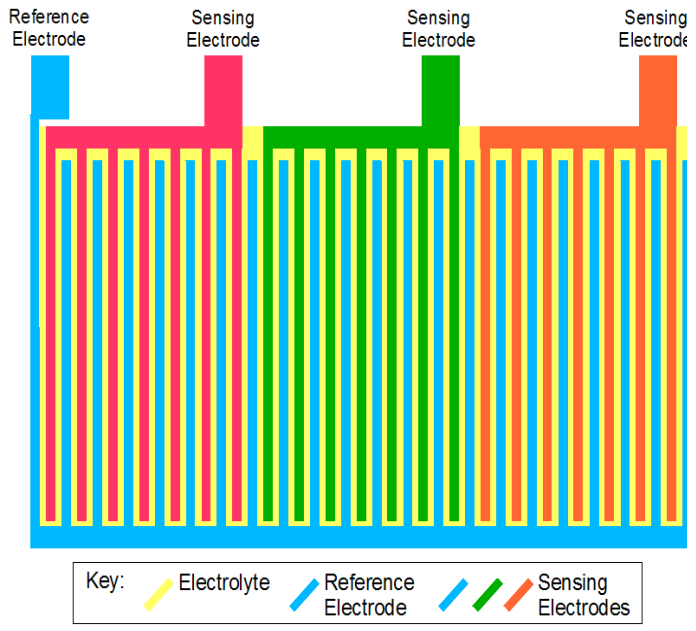

Fig. 2.1-3 Schematic of Triple Element Thin Film Sensor

The design parameters include: response speed, sensitivity, dynamic range, short and long-term stability, resolution, selectivity, operating ambient conditions, operating lifetime, output format, size, weight, and cost. Material selection for the microsensor is dependent on sensor performance characteristics.

\subsection{Sensor Selectivity}

A key advantage of voltammetry for the AMRDEC research and development project is that the selectivity can be increased via varying several parameters, as well as using sensor arrays. The sensor selectivity is dependent on the sensor material. Electrochemical sensors are not naturally selective and normally cannot clearly identify an unknown gas or mixture of gases. In voltammetric methods, selectivity may be enhanced relative to passive electrochemical methods (due to the forcing of the chemical system by an applied potential); however, it still falls short of allowing for adequate detection of a complex gaseous mixture, such as explosives. An alternative to this dilemma is to build an array of sensors, each with a different material that yields unique responses to a given analyte. If analytes are present at too dissimilar concentrations, high signal-to-noise ratio are required in order to discern the sought-after signal from noise originating from the larger concentrations of interferents. Raw sensor array data may be analyzed by chemometric 
methods such as principal component analysis or neural network methods. ${ }^{12}$ In order to change the response to a given analyte, the sensor chemistry must be altered or filters must be used.

In most electrochemical based sensors, the electrolyte contains a set of dissolved chemicals which react together, while ions and electrons migrate after an electrical potential is applied. ${ }^{13}$ In the proposed voltammetric sensor technology, the only current being conducted across the electrolyte is carried by oxygen anions, O2-. Rather than reactions taking place inside the electrolyte, all of the chemical transformations happen heterogeneously at the electrodes. Due to the porosity of the electrode, the analyte may contact both the electrode and the electrolyte simultaneously; this interaction is referred to as the triphasic boundary. Figure 2.2-1 schematically illustrates this boundary.

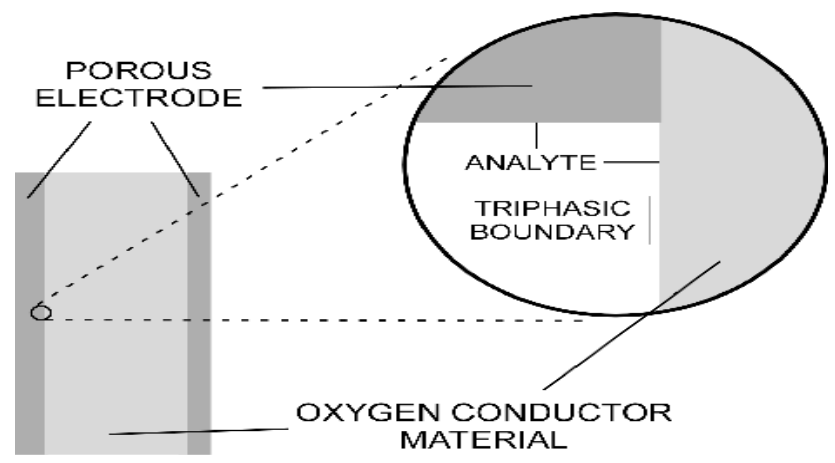

Fig. 2.2-1. Schematic of Triphasic Boundary

The i-V curve, voltammogram, resulting from the triphasic boundary interaction will therefore depend on the specific analyte and its response to the chemical composition of the electrode and electrolyte material. To alter the reaction, chemical signature, either the electrode or electrolyte must be changed. Computational models could be used to predict the various chemical signatures resulting from the interchange of materials, but for this effort, a more cost efficient method of trial-and-error was utilized.

As previously stated, the only current being conducted across the electrolyte of the AMRDEC thin-film microsensor, is that being carried by oxygen anions $\mathrm{O}^{-2}$. Therefore, the material for the electrolyte must be an excellent oxygen conductor. Fig. 2.2-2 presents the conductivity vs. temperature curves for several well-known oxygen conductors. ${ }^{14,15}$ From this chart, a subset of materials are selected based on cost and accessibility. The final selection for the microsensor is YSZ (yttria-stabilized zirconia) and WBO (tungsten-stabilized bismuth oxide), which are both successfully used to detect $\mathrm{CO}, \mathrm{CO}_{2}$, humidity, and various small organic molecules in the thick-film sensor. 


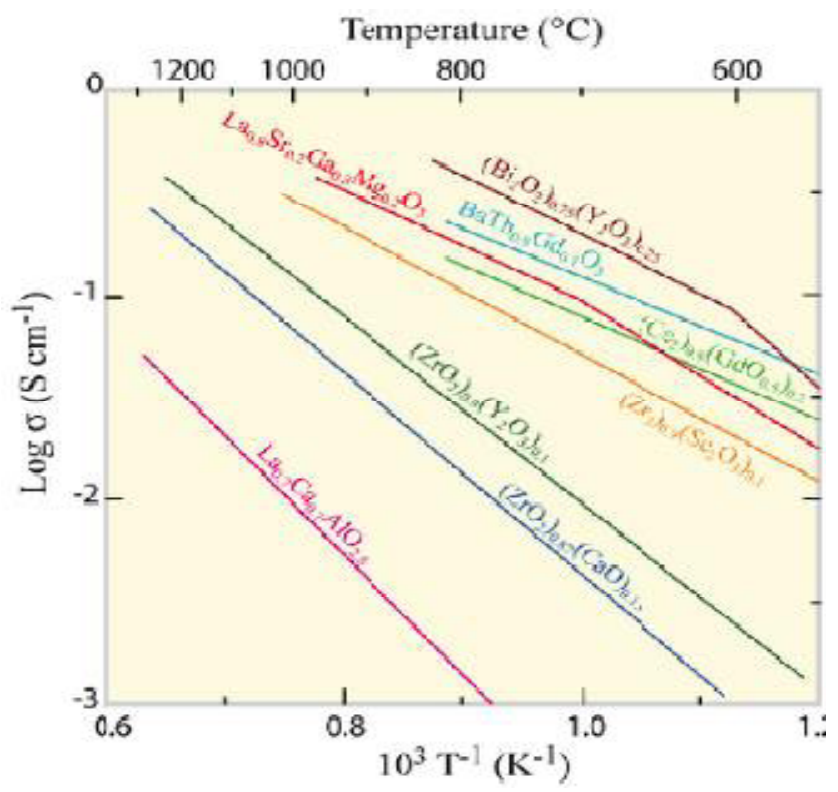

Fig. 2.2-2 (Ref.) Graph of Conductivity vs. Temperature of

The overall selectivity of the microsensor is increased by adding semipermeable membranes to the air-electrolyte interface or the electrolyte-electrode interface as shown in Fig. 2.2-3. Microporous and homogenous materials are two types of common air-electrolyte membrane materials. ${ }^{16}$ Microporous membranes are typically about $0.1 \mathrm{~mm}$ thick. Homogenous films are solid substances through which the analyte gas passes by diffusing. Homogenous films are much thinner than the microporous films, aiding in the expeditious transfer of gas and overall sensor response. The membranes may also be used to filter external environmental conditions or interfering chemical species prior to the interaction of these external negative stimuli with the sensor.

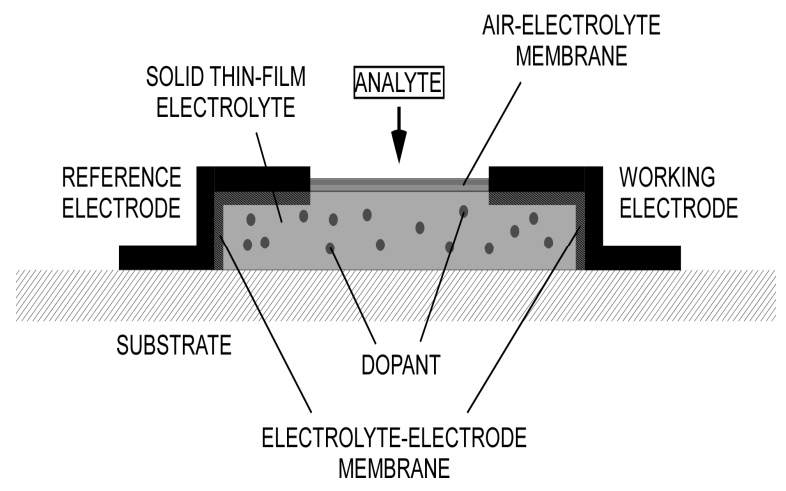

Fig. 2.2-3 Schematic of a Coplanar Microsensor 
Another method to increase the selectivity of the sensor is to dope or implant the electrolyte material with different species utilizing ion implantation. The depth and the quantity of the dopants are easily controlled but not predicted without computational modeling. For this effort, a more cost efficient trial and error method was utilized to characterize the response of dopants with the electrolyte. A schematic of the thick/thin film electrochemical cell with dopants is shown in Fig. 2.2-4.

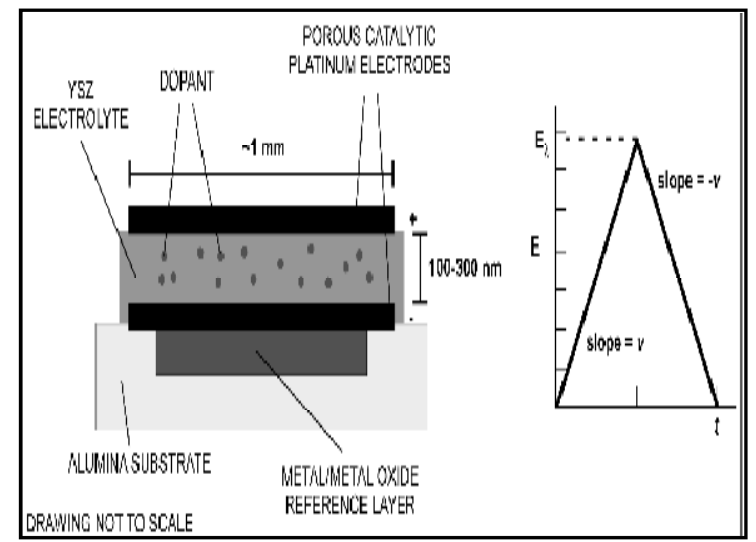

Fig 2.2-4 Schematic of Thick/Thin Film Electrochemical Cell with Dopants

\subsection{Sensor Fabrication and Mounting}

The thin-film sensors are fabricated on 3" silicon wafers using a multi-step process plan developed by Dr. Koy Cook of Alabama A\&M University. The process consists of wafer preparation and oxidation, photolithography, sputtering, etching, diffusion, and other conventional fabrication techniques at submicron dimensions. Most of the fabrication steps are performed using the fabrication facilities at Redstone Arsenal, with the remainder performed in the Clean Room at Alabama A\&M University. An indication of the actual size of the sensor is shown on a dime in Fig. 2.3-1 (a). A magnified image of the sensor in a microarray configuration is shown in Fig. 2.3-1 (b). Future design generations will reduce the size of the microarray and package the sensor readout electronics on one chip for system-on-a-chip applications.

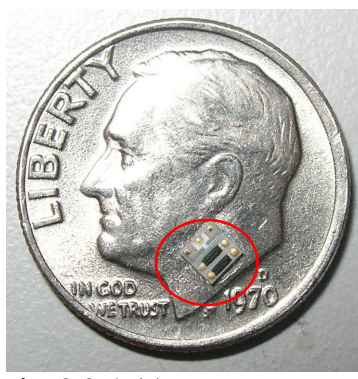

Fig. 2.3-1 (a) Mount prepared sensor on a dime.

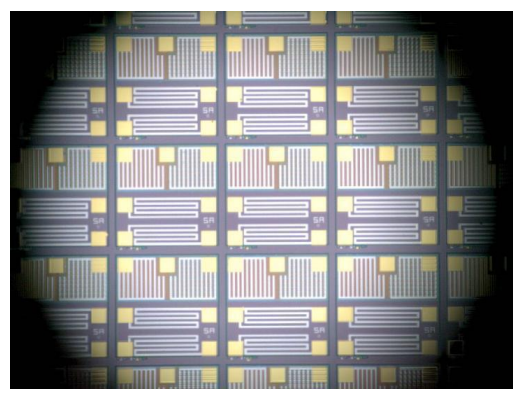

Fig. 2.3-1 (b) MicroArray of Sensors on a Silicon Substrate 
A single sensor is packaged in a standard TO-5 package. The TO-5 package is selected due to the accessibility of parts and the minimal cost of the parts. Fig. 2.3-2 (a) is a picture of the standard TO-5 part, with header and cap. The cap is mounted on the header. Fig. 2.3-2 (b) is an image of the voltammetric sensor mounted inside of the TO-5 cap.

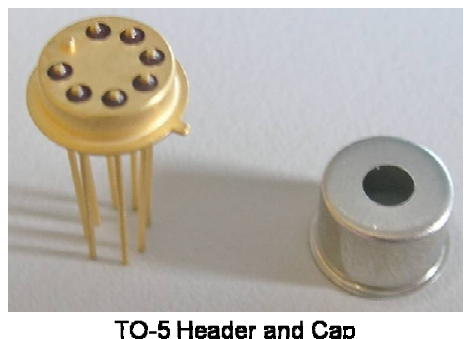

Fig. 2.3-2 (a) Standard TO-5 package

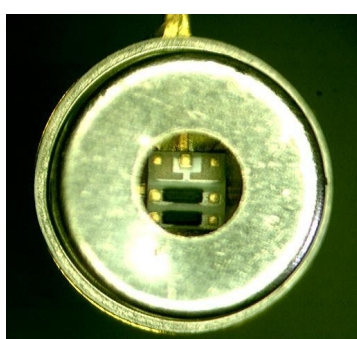

Fig. 2.3-2 (b) Sensor Mounted in TO-5 Cap

\subsection{Hardware Interface}

The voltammetric sensors function by applying a time-varying potential difference between the reference electrode and each of the working electrodes (as previously shown in Fig. 2.2-3), producing an electrochemical reaction-spectrumresponse. Each electro-active component chemical species in the target sample reacts with the electrochemical cells at characteristic reaction potentials that depend on the gas and the material composition of the electrochemical cells, resulting in current modulation through the cell. A potentiostat amplifies the current to a level that can be measured and captured by the A/D (analog to digital) and D/A (digital to analog) converters of the data acquisition circuitry (Fig. 2.41). This formatted data is recorded for processing as the chemical signature and ported to a ruggedized PC equipped with a National Instruments data acquisition card. The data acquisition/system control equipment is driven by software written in MatLab. The software has the ability to compare the characteristic voltammograms of the detected chemical species with a library of voltammograms of chemical species and environmental conditions.

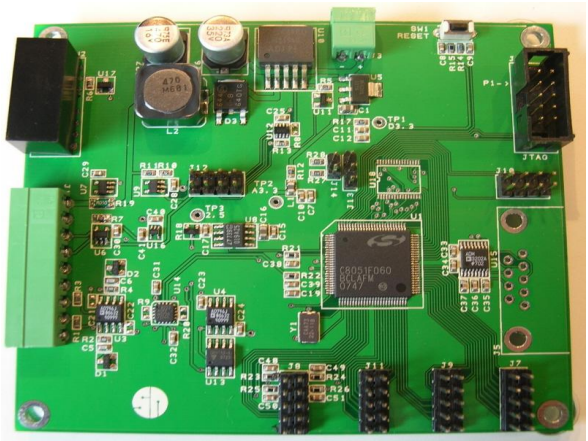

Fig. 2.4-1. Integrated Electronics

\subsection{Packaging of the Voltammetry System}

For normal atmospheric environments, the sensors and electronics are housed in a Teflon, acrylonitrile-butadienestyrene resin (Reference Fig. 2.5-1). Alternatively, application-specific ceramic housings can be substituted for extreme environments. Die-stamped stainless steel mesh cages are being evaluated as universal packages for common commercial applications. Cases are designed to protect the sensing element and to allow a controlled flow of air to be pumped across the sensor surface. If flow rates exceed $3.5 \mathrm{Lpm}$, internal baffles must be introduced to prevent convective cooling that can introduce measurement error. 


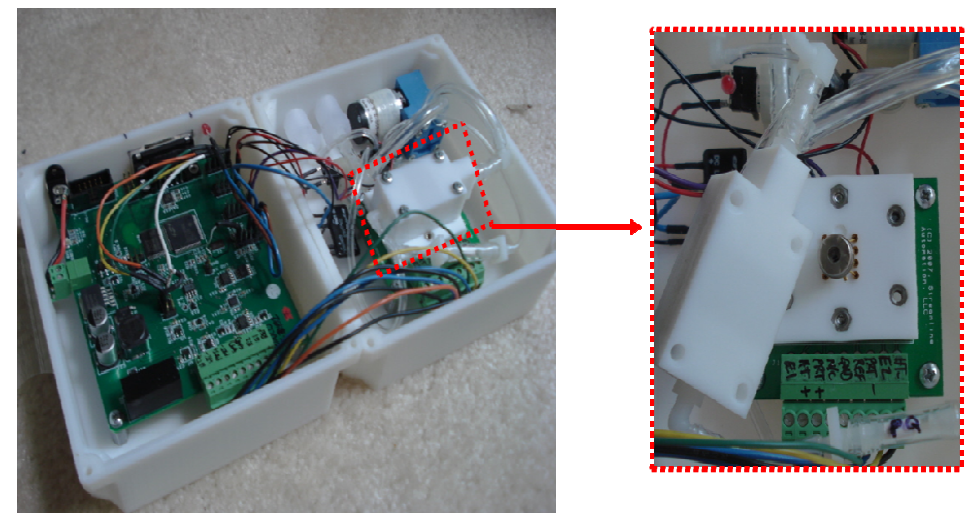

Fig. 2.5-1. Teflon Packaged Sensors and Electronics

\subsection{Technical Challenges}

Several technical issues requiring further research to form an effective solution to chemical species detection utilizing a voltammetric sensor are as follows:

1) Models to predict sensor selectivity to various dopants is required, but modeling can be costly and time consuming.

2) Controlling the sensor temperature and operational temperature is a design concern.

3) Developing the library of voltammograms to ensure proper filtering of undesired species.

4) Reducing the suite of sensor readout and data acquisition electronics to a low power, lightweight design in order to minimize the amount of additional payload required for unmanned vehicle mounting.

5) Developing wireless capabilities for unmanned vehicle configurations.

6) The sensing elements will be exposed on the wing surface of a UAV operating in a variety of environments that include dust, sand, and rain. The potential for damage/wear must be assessed, and protective coatings be developed, if necessary.

\section{INTEGRATION OF VOLTAMMETRY-BASED SENSOR SYSTEMS ON UNMANNED VEHICLES}

One of the main objectives of this research is to develop a chemical sensor array that can be used on small uninhabited aerial vehicles (UAVs), as well as ground vehicles. The first desire is to fabricate the sensor on a flexible substrate that can be readily mounted on and conformed to the wing of the UAV (as shown in Fig. 3.0-1). The advantage of this approach include the concept that the usable portions of the wing surface represent a potential surface area that is much larger than could be achieved in the normal UAV payload volume. Another advantage is that chemical sensors should benefit from increased surface area by yielding increased sensitivity. 


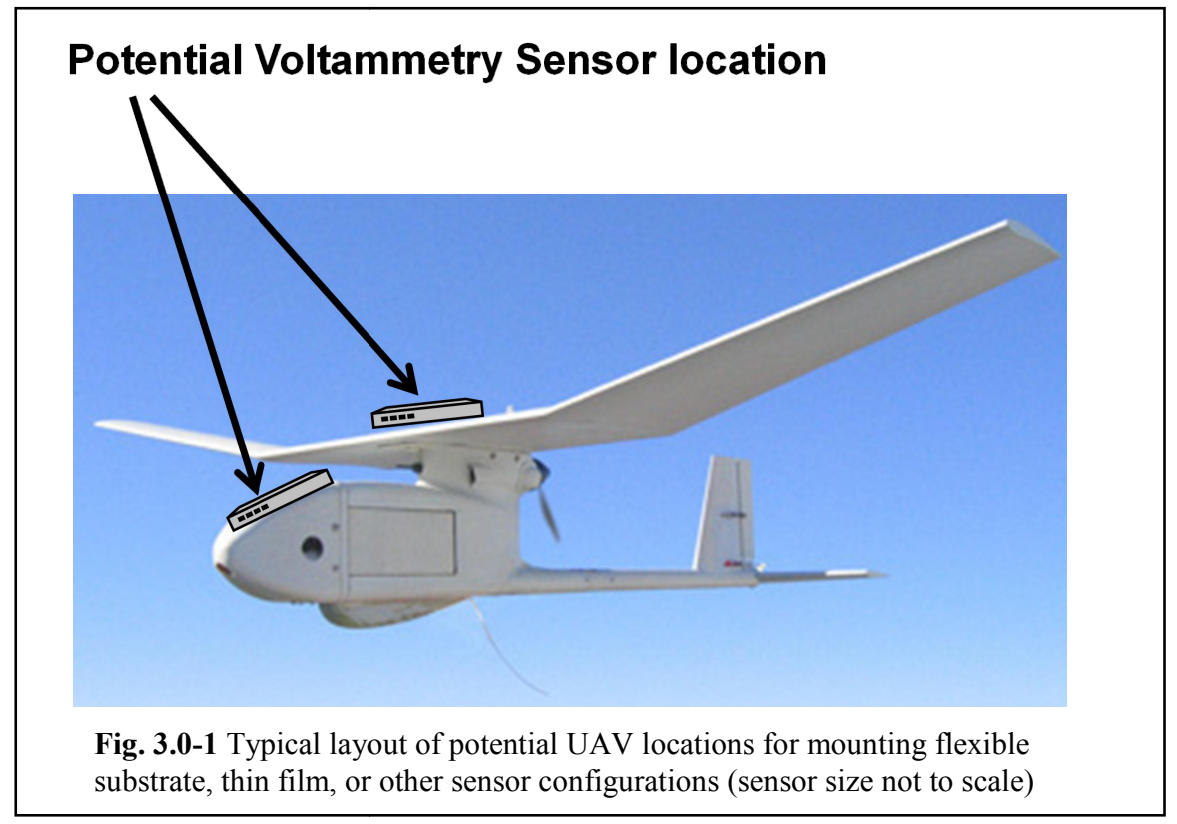

For the scenario previously shown in Fig. 3.0-1 (above), a technique must be developed for precisely positioning and holding the sensor. The expectation is that the mounting of the senor (especially in and around the UAV's nose area) will not interfere with any other payload that is normally added to the UAV. Since the weight of the sensor is expected to be small, the aerodynamics of the UAV is not expected to be impacted.

As part of additional efforts to determine potential locations and orientations for mounting the sensor, the authors communicated with another AMRDEC team at the Small UAV (SUAV) Laboratory of the System Simulation and Development Directorate. The SUAV Laboratory has the assignment of supporting the development of a chemical sensing payload for the Unmanned Aerial Systems (UAS) Project Office's uninhabited aircraft. Other members of the SUAV Laboratory team encompass Johns Hopkins University Applied Physics Laboratory and Aero Vironment. Although the results are not presented in this paper, the SUAV Laboratory team performed a ground electromagnetic impulse (EMI) test (for safety precaution), followed by an in-flight test with a functional chemical sensor payload. In association with the added weight of the sensor payload, the SUAV team likewise investigated UAV wing extensions in order to obtain an added lift capacity and to reduce the impact velocity generated during the UAV's deep stall recovery.

The authors' efforts were enhanced by performing an assessment of mounting and sensor orientation scenarios similar to those investigated by the SUAV Laboratory. Three additional approaches are shown in Fig 3.0-2 and labeled as A-C. 
The first scenario (Location "A") shows the sensor orientated and mounted parallel with the inline direction of the UAV nose. In the second scenario (Location "B"), the smaller side of the sensor is mounted with the longer axis oriented perpendicular to the UAV nose. In the final scenario (Location " $C$ "), the larger side of the sensor is mounted with the longer axis oriented perpendicular to the UAV nose.

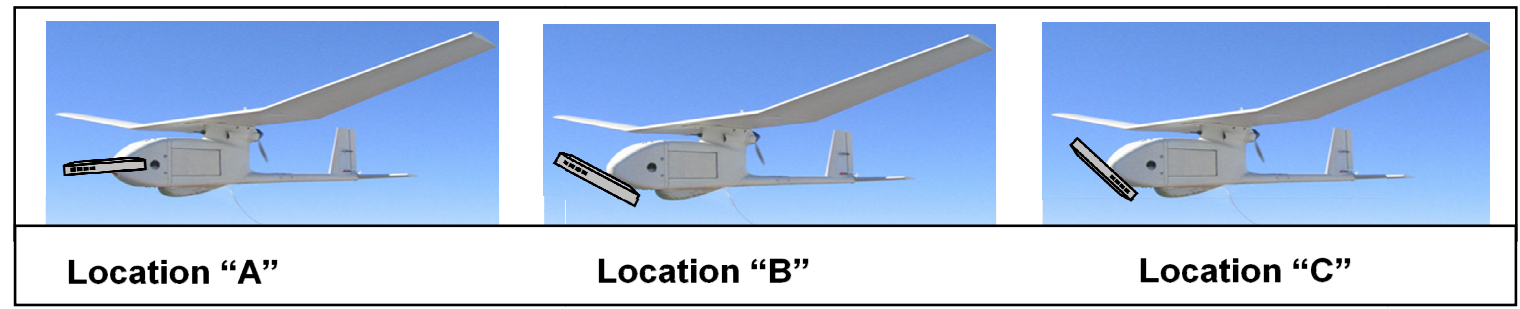

Fig. 3.0-2 Alternative Mounting Orientations for the Sensor on the UAV Nose Area (sensor size and exact orientations are not to scale)

When considering sensor mounting and orientations configurations, one important consideration is the flexibility of the sensing element materials to conform to the wing or other UAV surface contours.

Other considerations include the sensor's ability to withstand dust, sand, and rain while being mounted on wing, nose, or other surfaces of the chosen UAV. Attention must be given to the potential need for development of damage protection by incorporating protective coatings or other components.

All payload devices are modular in design to enhance mission flexibility, and to allow rapid installation/removal in a field environment using no tools. Also, these payloads are modular in nature so they can be added and removed as required for specific missions.

\section{TEST RESULTS}

\subsection{Test Setup}

The sensor system was tested to measure the sensitivity to undiluted trace amounts of ammonia, mustard gas stimulant (CEES), VX stimulant (Diazonin), GB, GD stimulant (DMMP), nitrogen tetraoxide (NTO), monomethyl hydrazine $(\mathrm{MMH})$, and hydrazine. The sensors are enclosed in a Pyrex glass enclosure, 3 liter three-neck flask. Gases from the stock cylinders are injected into the septum and directed through precision flow meters into the glass chamber, as shown in Fig. 4.1-1. The chamber is flushed with air, and the sensor readings are measured and recorded.

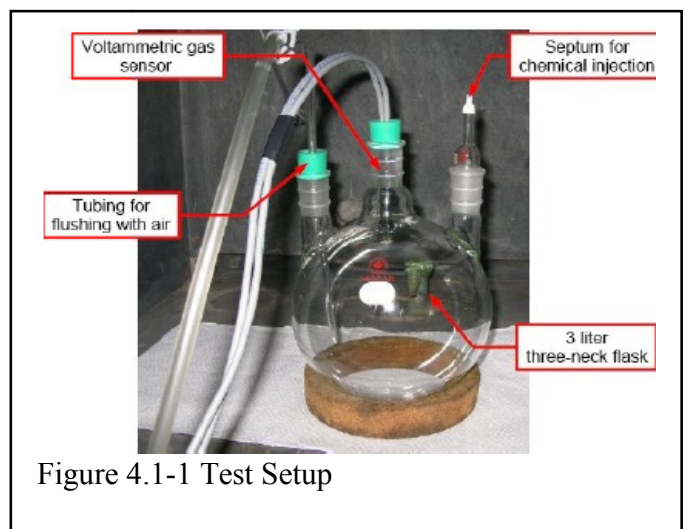




\subsection{Sensor Response}

The response/output of the voltammetry sensor system is complex waveforms containing a large amount of data, referred to as voltammograms. The voltammogram depends on the type of electro-active gaseous components present in the target analyte. The chemical signature resulting from the voltammogram produces the identity and quantity of the present chemical species that interacted with the sensor's electrochemical cell. The advantage to this type of sensing method over single sample point responses is the significant amount of information on the rate-of-change and intervariable relationships produced by time and parameter based features on the voltammograms. These response features also aid in the identification of signal trends. The occurrence of chemical reactivity is determined from the calculation and comparison of the target analyte chemical signature to the background air signature. Pattern recognition tools and neural network analysis are used to extract the analog values representing the chemical composition of the target analytes.

An example of the raw data generated voltammogram for ammonia and the general regression neural network classification results of sensor testing with ammonia $\left(\mathrm{NH}_{3}\right)$ concentrations as low as 178 ppbv is shown in Fig. 4.2-1 (a) and (b).

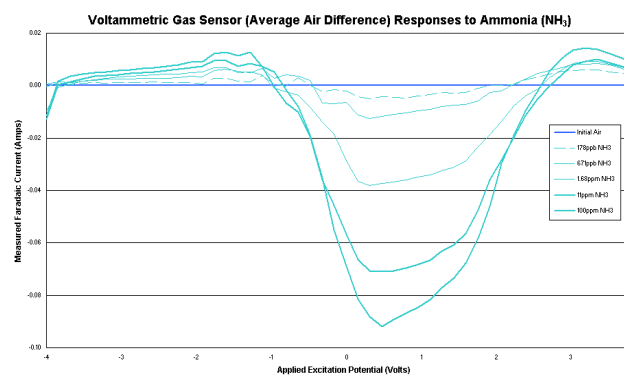

Fig. 4.2-1 (a) Raw Data of Sensor Response to Ammonia

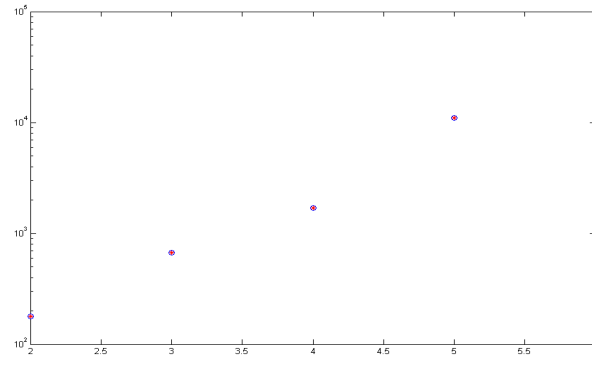

Fig. 4.2-1 (b) GRNN Classification Result for Ammonia

\subsection{Test Results}

A single element sensor is exposed to ammonia from lowest to highest concentration, followed by a background air sample. The data from the sensor's reaction to ammonia was previously shown in Fig. 4.2-1 (a) and additional data is presented in Fig. 4.3-1. The voltammogram (Fig. 4.3-1) shows +/- 2 Volt sweep limits, and focuses on the difference between the ammonia responses and the background air responses. Each sweep occurred over a 1 second interval. Measurements were taken from 0-109 ppm of ammonia. As the measurement cycle is repeated, each successive peak narrowed, representing a function of less and less other species present to react. An increase in concentration of the ammonia is depicted on the graph where each successive peak rose in amplitude. Each successive peak in the chemical signature also shifts toward a stable dissociation potential.

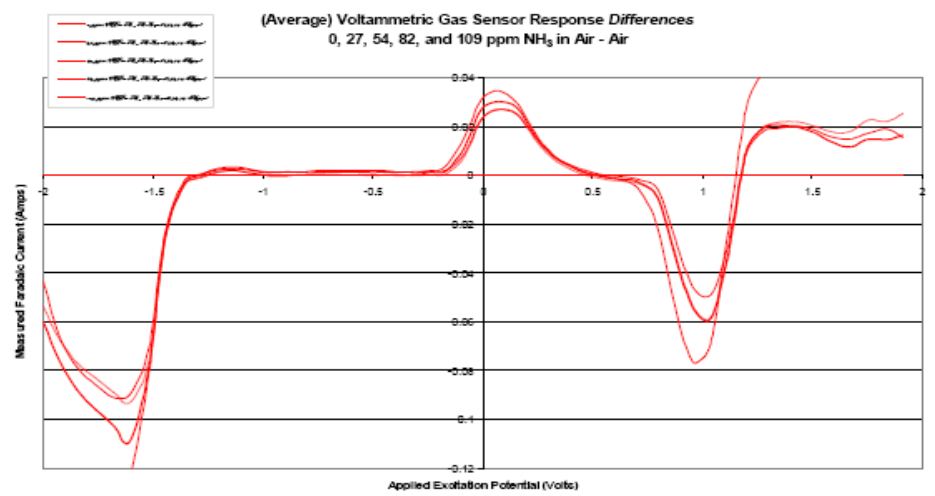

Fig. 4.3-1 Raw data of Sensor Results to Ammonia 
The sensing element is also exposed to common rocket motor out-gassing toxic compounds. ${ }^{17}$ The spectral response of the sensing element is analyzed using common spectral analysis techniques to identify the chemical at various concentrations in the gas sample. Curves typifying the sensitivities of the selected gas samples [.05 ppm of hydrazine, $1.5 \mathrm{ppm}$ of monomethyl hydrazine (MMH), and $6 \mathrm{ppm}$ of nitrogen tetroxide (NTO)] are shown in Figure 4.3-2. The raw voltage sensor relates the applied voltage to the measured current passing through the electrochemical cell. As the voltage sensor output is analyzed, the location of the peaks along the voltage axis indicates which species are present, and the magnitude of peaks reflects the concentrations of each component. This information is used to identify and quantify chemical species in the environment.

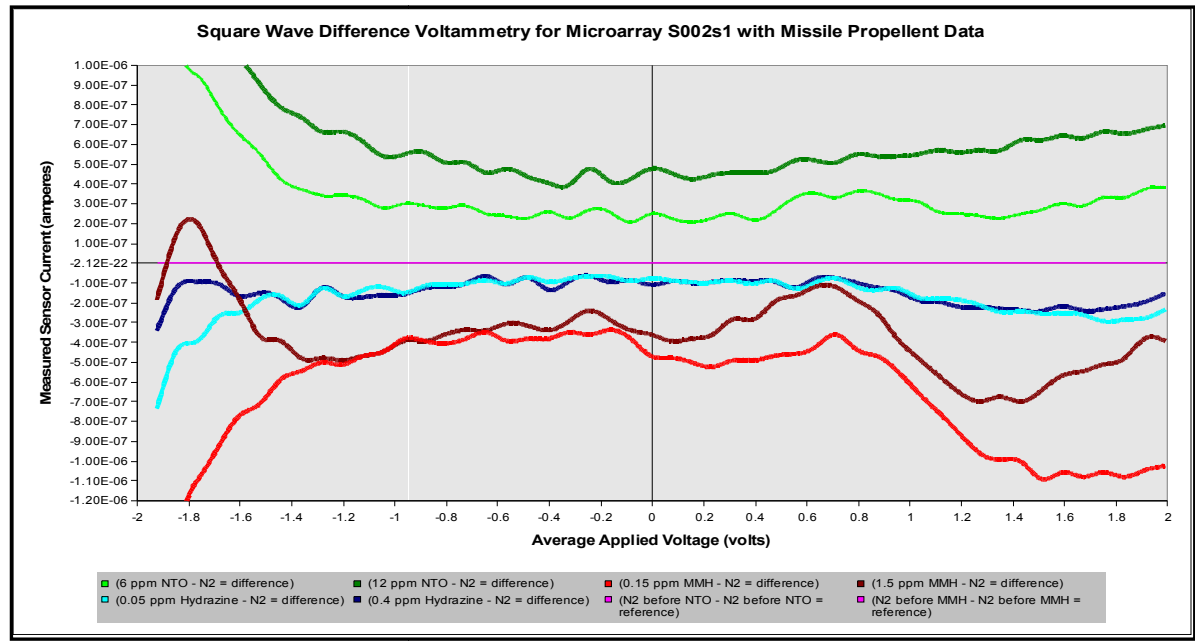

Fig. 4.3-2 Graph of Thick Film Gas Sensor Sensitivities to Rocket Out-gassing Chemicals

A summary of the chemicals tested and concentration levels are shown in Table 4.3-1.

Table 4.3-1 Results from Detected Chemical Agents

\begin{tabular}{|c|c|c|c|} 
TIC representative & $\mathrm{H}, \mathrm{HD}$ simulant & VX simulant & $\mathrm{GB}, \mathrm{GD}$ simulant \\
\hline$\Lambda$ mmonia & $\mathrm{CFFS}$ & Diazinon & DMMP \\
$\mathrm{NH}_{3}$ & $\mathrm{CCH}_{2} \mathrm{CH}_{2} \mathrm{SCH}_{2} \mathrm{CH}_{3}$ & $\mathrm{C}_{12} \mathrm{H}_{21} \mathrm{~N}_{2} \mathrm{O}_{3} \mathrm{PS}$ & $\mathrm{CH}_{3} \mathrm{PO}_{\mathbf{3}}\left(\mathrm{CCH}_{3}\right)_{2}$ \\
\hline $178 \mathrm{ppbv}-$ & $46 \mathrm{pptv}-$ & $>1 \mathrm{pptv}-$ & $13 \mathrm{ppbv}-$ \\
$100 \mathrm{ppmv}$ & $517 \mathrm{ppbv}$ & $682 \mathrm{pptv}$ & $5856 \mathrm{ppbv}$ \\
\hline
\end{tabular}

\begin{tabular}{|c|c|c|}
\hline Chemical & Low concentration & High concentration \\
\hline NTO (Nitrogen tetraoxide) & $6 \mathrm{ppm}(6,000 \mathrm{ppb})$ & $12 \mathrm{ppm}(12,000 \mathrm{ppb})$ \\
\hline $\begin{array}{c}\text { MMH (Monomethyl } \\
\text { hydrazine) } \\
\text { Hydrazine }\end{array}$ & $0.15 \mathrm{ppm}(150 \mathrm{ppb})$ & $1.5 \mathrm{ppm}(1,500 \mathrm{ppb})$ \\
& $0.05 \mathrm{ppm}(50 \mathrm{ppb})$ & $0.4 \mathrm{ppm}(400 \mathrm{ppb})$ \\
\hline
\end{tabular}




\section{SUMMARY}

A synopsis of the AMRDEC research and development project has been presented in this paper for integrating sensor array systems onto uninhabited vehicles. The results outline information relative to the development of nano-based chemical sensor array systems for both ground and airborne uninhabited vehicles. Voltammetry-based and other sensors are used to detect and identify multiple analytes from explosive devices and chemical warfare agents. The thick-film voltammetric sensor technology developed by Argonne National Laboratory is used as a baseline to develop the thick/thin film chemical agent detector that is presented in this paper. The thick/thin film sensor is fabricated, prototyped with integrated electronics and laboratory tested. In addition, the thin-film voltammetric sensor is designed and fabricated. Laboratory testing is currently underway. Preliminary results indicate that the thick/thin film-based sensor system react to extremely small amounts of explosives and chemical warfare agents. The technical challenges include modeling sensor behavior, controlling the internal sensor temperature, miniaturizing the electronics, and ruggedizing the sensor system for UAV mounting. Future efforts will concentrate on field testing the current thin-film sensor system, integrating GPS technology, and reconfiguring the sensor system to conform to and be surface-mounted to manned or uninhabited vehicles.

\section{ACKNOWLEDGEMENTS}

The authors wish to thank the outstanding contributions of physics and engineering professors at Alabama Agricultural and Mechanical University (AAMU) for their outstanding contributions for sensor arrays for prognostic and diagnostic systems under Contract W31P4Q-05-C-0262. Acknowledgement is also given to Streamline Automation for their subcontractor support of AAMU in the development of voltammetry based sensors for missile health applications and chemical agent detection. The authors would like to especially thank the AMRDEC team at the Small UAV (SUAV) Laboratory for providing U AV mounting data. Thanks to the AMRDEC MEMS and Nanotechnology Working Group, for sharing their expertise in the identification of performance requirements issues associated with weapons health monitoring.

\section{REFERENCES}

[1] Star, A., Bradley, K., Gabriel, J. P., and Gruner, G., "Nano-Electronic Sensors: Chemical Detection Using Carbon Nanotubes," Polymeric Materials: Science \& Engineering, 89, 204, (2003).

[2] Tans, S., Verschueren, A., and Dekker, C., "Room-temperature Transistor based on a Single Carbon Nanotube," Nature 393, 49-52 (1998).

[3] Collins, P. G., Bradley, K., Ishigami, M., and Zettl, A., "Extreme Oxygen Sensitivity of Electronic Properties of Carbon Nanotubes," Science 287, 1801 (2000).

[4] Hammond, M., "General Atomics Smart Microsensors - FY05 Shipboard Fire Tests Results," Contract\#NRL/MR/6180-05-8928, ( 2005).

[5] Siegel, J., "Joint Chemical Agent Detector: One Detector Providing Multiple Capabilities to the Joint Warfighter," Chem-Bio Defense Quarterly, 5, 5-7, 2008

[6] Singer, N., "Ultralight Device Analyzes Gases Immediately. Flying SnifferSTAR may aid civilians and U. S. Military", Sandia National Laboratories Press Release, Jan. 23, 2003.

[7] Sanders, J.M., "Sensing Danger," Research Horizons, Homeland Security, Fall 2004.

[8] Vogt, M., Shoemaker, E. and Turner, T., "A Trainable Cermet Gas Microsensor Technology using Cyclic Voltammetry and Neural Networks," Sensors and Actuators B 35-36, 370-376 (1996).

[9] Kincade, K., et. al., "Optoelectronic applications: nanophotonics - an 'old' technique finds new life in the nano world," http://www.laserfocusworld.com/articles/274732, 1 (2007).

[10] Currie, J., Essalik, A. and Marusic, J. "Micromachined thin film solid state electrochemical CO2, NO and SO2 gas sensors," Sensors and Actuators B: Chemical 59, 235-241 (1999). 
[11] Dubbe, A., Nafe, H. and Aklinger, F., "Patterned micro electrodes on yttria-stabilized zirconia thin films," [Book of Abstracts, 4th International Symposium on Electrochemical Micro- and Nanosystem Technology], Dusseldorf, Germany, 15-20 (2002).

[12] Sharma, A., Saafi, M., and Heidary, K., “Alabama A\&M University Sensor Research," Contract \# W31P4Q-05-C0262, (2007, 2008).

[13] Reich, A. J., "Sensor Technology," http://www.streamlineautomation.biz/sensor_technology.html, 1 (2008).

[14] Skinner, S. and Kilner, J. "Oxygen ion conductors." Materials Today, 30 (2003).

[15]DoITPoMS, D.o.M.S.a.M., University of Cambridge, "Fuel cell electrolytes," http://www.doitpoms.ac.uk/tlplib/fuelcells/sofc electrolyte.php, University of Cambridge, (2008).

[16] Skoog, D., Holler, F. and Nieman, T., [Principles of Instrumental Analysis, Edn. 5th.], Harcourt Brace \& Company, Orlando, FL, (1998).

[17] Brantley, C. L., Ruffin, P. B. and Edwards, E., "Innovative smart microsensors for Army weaponry applications," Proc. SPIE 6931, 6931-1 (2008). 